\title{
ABOUT THE CONTRIBUTORS
}

Yot Amornkitvikai, Lecturer, College of Population Studies, Chulalongkorn University

Kala Anandarajah, Head, Competition \& Antitrust and Trade Lead Partner, Corporate Governance

Kathleen Azali, Founder of C2O library \& collabtive, Surabaya

Sanchita Basu Das, Economist, Asian Development Bank (ADB)

Yose Rizal Damuri, Head of the Department of Economics, Center for Strategic and International Studies

Cassey Lee, Senior Fellow, ISEAS - Yusof Ishak Institute, Singapore

Eileen Lee, staff member, Policy and Markets Division, Competition and Consumer Commission of Singapore (CCCS), 2016-18

Siwage Dharma Negara, Senior Fellow, ISEAS - Yusof Ishak Institute, Singapore

Nguyen Thi Hong Van, Lecturer, Electronic Commerce Department, Foreign Trade University, Hanoi, Vietnam

Nguyen Van Thoan, Associate Professor, Electronic Commerce Department, Foreign Trade University, Hanoi, Vietnam 
Lip Hang Poh, staff member, Policy and Markets Division, Competition and Consumer Commission of Singapore (CCCS), 2016-17

Shanti Aubren T. Prado, Policy Research Officer IV, Philippine Competition Commission

Meg L. Reganon, Senior Technical Assistant, Philippine Competition Commission

Tanya Tang, Partner (Chief Economic and Policy Advisor), Rajah \& Tann Singapore LLP

Jiraporn Tangpoolcharoen, Specialist, Office of Strategy, Electronic Transactions Development Agency (Public Organization) (ETDA)

Tham Siew Yean, Senior Fellow, ISEAS - Yusof Ishak Institute, Singapore

Zheng Xi, Associate, Rajah \& Tann Asia Singapore LLP 
